\title{
Multi-Level Screening Identification for Lunar Impact Craters*
}

\author{
Jiaheng Chen ${ }^{1, a}$, Zhankai $\mathrm{Li}^{1, \mathrm{~b}}$ and Xiaolin Tian ${ }^{1,2, \mathrm{c}}$ \\ ${ }^{1}$ Faculty of Information Technology, Macau University of Science and Technology, Macao 999078; \\ ${ }^{2}$ Lunar and Planetary Science Laboratory/Space Science Institute, Macau University of Science and \\ Technology, Macao 999078. \\ achenjiaheng1992@126.com, bjanlzk@126.com, 'x xltian@must.edu.mo
}

Keywords: Lunar impact crater, CCD, recognition algorithm, multi-level.

\begin{abstract}
This study proposes a Multi-Level Screening Identification algorithm for the automatic extraction and identification of impact craters based on CCD image. Considering of the comparatively low robustness of traditional algorithms, we design analytical hierarchy process in the new algorithm. First of all, we analyze the whole image, and tag the candidate regions. Next, make the different preprocessing for the different areas respectively. Then, identify the craters from the different area. Finally, the algorithm will check the result of identification automatically. To test the algorithm, we choose the image from the H010, SI and Crisiums. The proposed algorithm provides a good robustness in most of lunar areas.
\end{abstract}

\section{Introduction}

The moon is the nearest planet from the earth and all the time human want to explore it as the first target. Until 21th century, the Chang'e Project had started officially in our country after the ShenZhou VI and ShenZhou VII launched successfully. Lunar image processing is one of most important approach to the lunar research. Among them the topography and the landform of lunar are the key point to the history evolution research. Otherwise, lunar image processing and result represent the research standard today. Nowadays, lunar image processing technique still on the initial stage, most of works still rely on manual work. Nevertheless, manual work cannot satisfy the large number of planet terrain data. Therefore, researchers have tried to promote the technique of computer and analyses the data.

Several of craters are the main feature of the lunar terrain. Not only on the surface of lunar, but also Mars and any other planets have same kind of craters. Those craters have different sizes, depth and shapes; they are evidence of planets history [3]. It is no deny that these craters provide plenty of useful data about the age of planet.

It is an important technology for lunar research that a large amount of data calculation and analysis by computer. Therefore, there are increasingly researchers join in this technic area. From the beginning, research method had changed from manual observation and identification to the huge amounts of automatic identification. From the source of data, lunar crater can be classified into two parts. Most of identification and analyze were based on CCD images, in lunar research we also call it LROC (Lunar Reconnaissance Orbiter Camera) images. These images usually have higher resolution than DEM data [6].

In this paper, we made algorithms which can identification impact craters only based on CCD image. Take advantage of the high resolution, our algorithm using multi-level screening to eliminate interference in image and accurate extract craters step by step.

\section{Multi-Level Screening Algorithm}

\subsection{The flowchart of Multi-Level Screening Algorithm}

From Fig. 1 we can have an overview of our algorithm. 


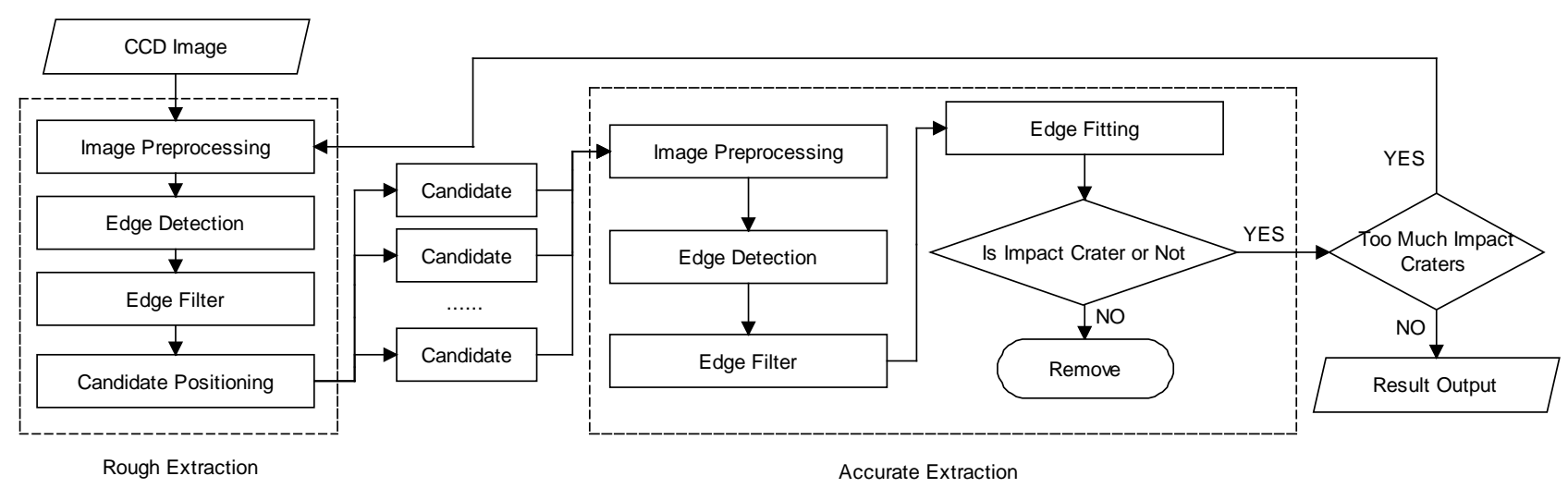

Fig.1 The flowchart of Multi-Level Screening Algorithm

The flowchart in Fig. 1 shows the new idea of craters identification. According to the result of experiment, the size of image directly affects the accuracy of the extraction and identification. Therefore, we use multi-level screening to extract the craters step by step.

\subsection{Edge Detection and Edge Filter}

Before the preliminary extraction of crater candidates, we should do the image preprocessing [4]. After image preprocessing, we begin to enter the rough extraction phases. First and foremost, we should extract the edges without noise and false edges. There are many kinds of edge extraction methods, such as Robert, Sobel, Prewitt and Canny operators [5]. Rugged surface and rocks on the lunar terrain has brought a lot of noise. The edge extraction algorithm must be adapted to the lunar surface characteristics and ignore the false point [10]. Through experimental comparison, Canny has better anti-interference performance. Therefore, we choose Canny operator.

Although Canny operator can ignore the most of noise edge, it still cannot avoid some of false edges [2]. Actually, some edges extracted by Canny are not false edges. Some edges generate by sun light shadow but we do not want them. In order to solve this problem, we filter the false edge through the illumination angle.

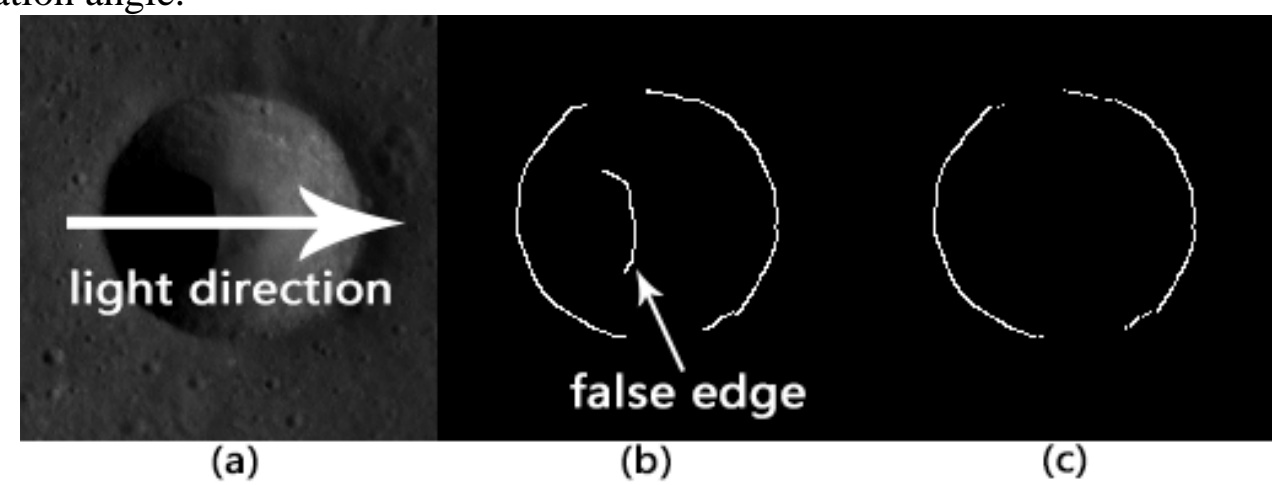

Fig. 2 Edge filtering algorithm. (a) Crater and light direction. (b) False edges in the edge extraction result. (c) The image after the false edges is removed.

Fig. 2 shows the effect of edge filtering algorithm. We detected each edge, calculate their light and shade direction through the illumination angle [6]. After these steps, most of false edges will be removed.

\subsection{Rough Extraction}

After several steps, we can try to extract the craters candidates. Craters are usually be fitted by the shape of circle or ellipse. Owing to this reason, circle fitting and ellipse fitting methods are usually worked for lunar craters extraction [1].

The Hough transform is a feature extraction technique used in image analysis. The purpose of the technique is to find imperfect instances of objects within a certain class of shapes by a voting procedure. This voting procedure is carried out in a parameter space, from which object candidates are obtained as local maxima in a so-called accumulator space that is explicitly constructed by the 
algorithm for computing the Hough transform [10]. The fitting algorithm we used is based on Hough transform which is called Hough circle fitting.

Calculated by Hough algorithm, the candidate regions which contained suspicious of impact craters will be tagged and extracted. As Fig. 3 shown below, the candidate regions will be extracted to the crater extraction phase [8].

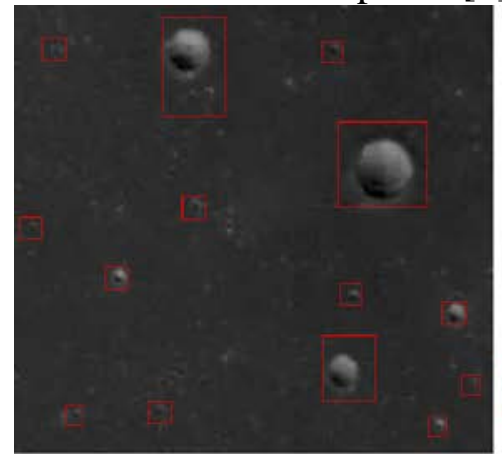

(a)

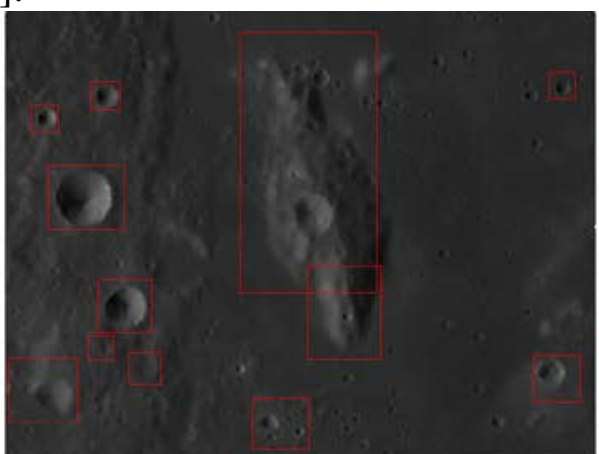

(b)

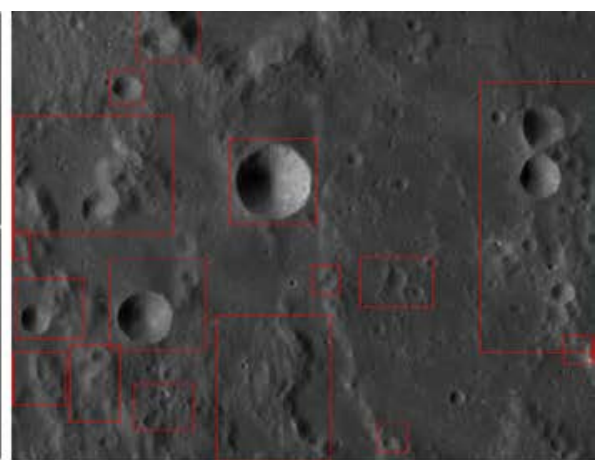

(c)

Fig. 3 Candidate regions extracted by rough extraction phases. (a) Lunar mare in SI. (b) Lunar mare and highland in H010. (c) Lunar highland in H010.

\subsection{Crater Extraction}

As the flowchart Fig. 1 shown above, the candidate regions will be extracted as new size image input to the crater extraction process. As we know, the image size and the number of information will affect the effect of image processing. Different area may interact with each other and reduce the recognition accuracy directly.

After rough extraction, each candidate regions will be adapted to their own image characteristic. This phases we call it crater extraction phases. There are several steps in crater extraction phase which can select threshold to get the better effect. In addition, different candidate regions have different contrast ratio and image size also affect the processing method and suitable threshold. Therefore, reduce searching range can help algorithm have a better performance on self-adaption [10].

In this part, some of processing steps are using the same algorithms as the first screening. The edge detection, edge filter and edge fitting algorithm still play an important role. Finally, the number of extracted craters will decide to output results or further select candidate regions.

\subsection{Crater Judgment}

After the several steps in the crater extraction phase, the last step is crater judgment. This step help remove some of false identification result. The principle of judgment is the light direction [7]. As Fig. 4 shown below, when we know the light direction, we can find their rule. Through this method we can remove some of false identification result.
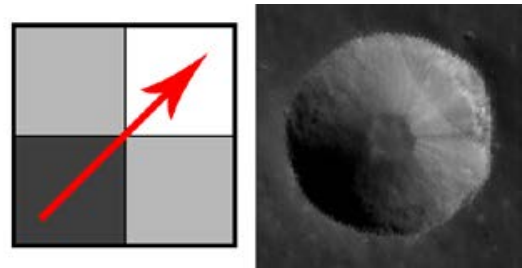

Fig. 4 Light direction and characteristic of craters

\section{Identification Result}

We try to identify the different parts of the terrain. In our experiments, we choose to extract the craters which radius more than 10 pixels. After the detection, we find this algorithm usually has a good result on lunar mare areas and some part of the flat highland areas. The identification effects have shown in Fig. 5. In Fig. 5, we can find the obvious impact craters recognition effect is good. Their extraction accuracy usually stays in a high level. However, the craters around 10 pixels show lower accuracy of identification. 


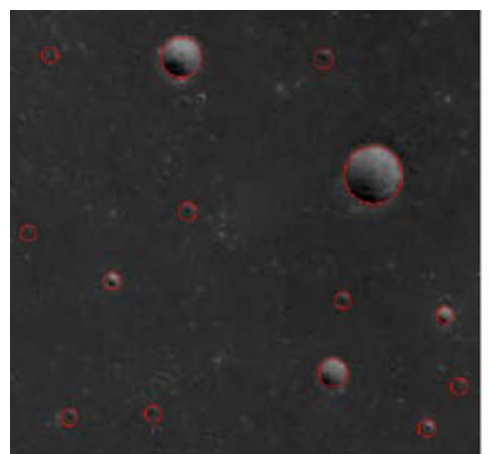

(a)

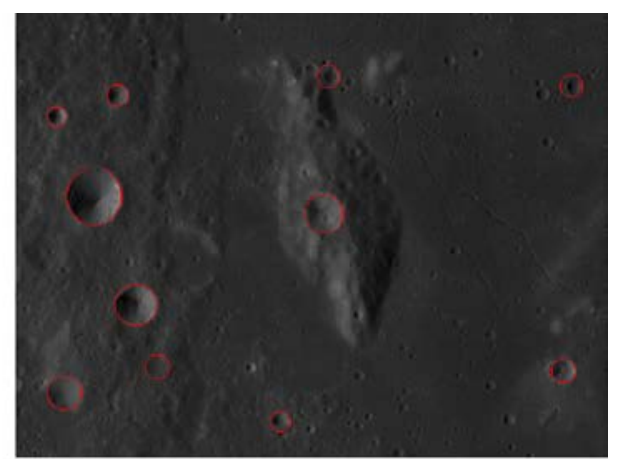

(b)

Fig. 5 Identification result in different area. (a) Lunar mare in SI. (b) Lunar mare and highland in H010.

Fig. 6 shows some part of lunar highland areas. Studies have shown that their high rugged terrain characteristic reduces the recognition rate. In Fig. 6, from (a) to (c) show that with the increase of terrain complexity, recognition rate is falling. The worst result in Fig. 6 (c) shows the algorithm cannot extract the right position.

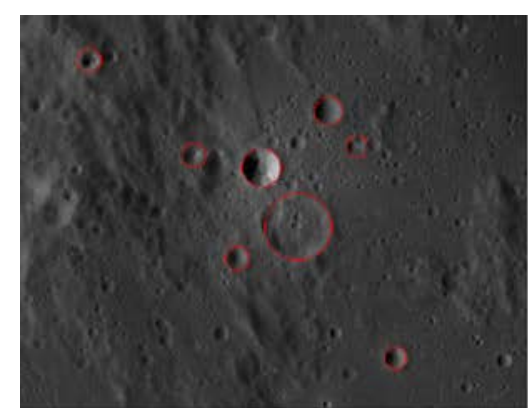

(a)

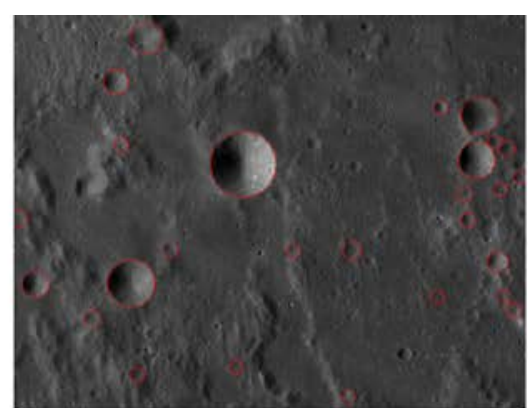

(b)

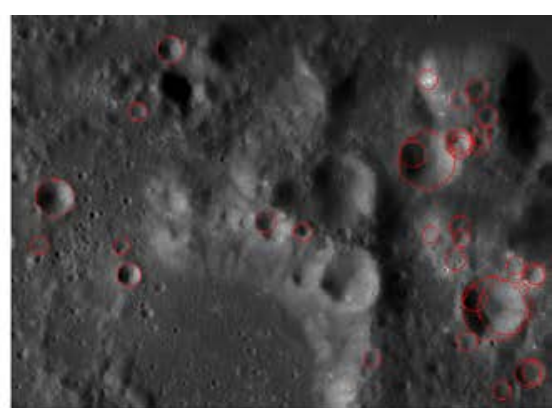

(c)

Fig. 6 Identification result in complex terrain. (a) Relatively flat terrain in H010. (b) The rugged terrain in H010. (c) More complicated terrain in Crisium.

Manual extractions were compared with automatic extraction in Table 1. Due to the limitations of the computer algorithm, we only extract the craters which radius more than 10 pixels. The false extractions also show the algorithm effect in different image. Obviously, we got better accuracy of automatic extraction in flat areas. The results in Fig. 5 show less error identification while Fig. 6 has higher error identification. With the growth of the image complexity, the false extraction also raised. On the whole, automatic extraction algorithm has a good performance on large scale craters extraction. However, false extraction always appeared in the identification of a small size craters.

Table 1 Three Scheme comparing (radius $>=10$ pixels)

\begin{tabular}{cccc}
\hline Fig. & $\begin{array}{c}\text { Manual } \\
\text { extraction }\end{array}$ & Automatic extraction & False extraction \\
\hline 5(a) & 12 & 14 & 2 \\
5(b) & 9 & 11 & 2 \\
6(a) & 10 & 8 & 1 \\
6(b) & 23 & 27 & 6 \\
6(c) & 28 & 34 & 14 \\
\hline
\end{tabular}

\section{Conclusion}

According to the identification result, we can have a conclusion:

In most of images, the algorithm shows a high accuracy on large scale craters. In flat areas, small scale crater still can be extracted and shows the high accuracy on it. However, as the image complexity increased, the extraction accuracy decreased. The worst result still appeared in lunar 
highland areas. Because of the complexity of terrain characteristic, our algorithm shows a lot of false extraction results.

Some deficiencies of our algorithm can be concluded as below.

(1) Cannot get a good performance on small size of craters identification.

(2) The process of choosing candidate regions still cannot easy distinguish lunar mare and highland areas. Increase the terrain recognition can improve the effect of algorithm.

(3) Low identification accuracy on complex terrain.

(4) Cannot get a good performance on blurred areas of high resolution images identification.

*This work is supported by the Science and Technology Development Fund of Macao (No.

059/2013/A2).

\section{References}

[1]. YUAN YueFeng, ZHU PeiMin, ZHAO Na, et al. Automatic identification of circular mare craters based on mathematical morphology. SCIENTIA SINICA Physica, Mechanica \& Astronomica. Vol.43 (2013) No. 3, p. 324-332.

[2]. DU Jun, MIAO Fang, LU Yuhang, et al. Research on appraisal of edge definition of impact craters. Computer Engineering and Applications, Vol. 49 (2013) No. 15, p. 179-183.

[3]. CHEN Ken, LIAO Ping, WANG Yuchuan, et al. Research on Digital Quantitative Classification Method of the Lunar Surface Spots. New Technology \& New Process. Vol. 1 (2015) No. 5, p.106-109.

[4]. Liu Yuxuan, Liu Jianjun, Mu LingLi, et al. A Review of Impact-Crater Detection. ASTRONOMICAL RESEARCH \& TECHNOLOGY. Vol. 9 (2012) No. 2, p. 203-212.

[5]. LUO Lei, WANG Xinyuan, Ji Wei, et al. Automated Detection of Lunar Craters Based on Chang'E-1 CCD Data. 2011 4th International Congress on Image and Signal Processing (CISP 2011). Shanghai, China, 15-17 October 2011, p. 883-887.

[6]. Zhizhong Kang, Zhongfei Luo, Teng Hu, et al. Automatic Extraction and Identification of Lunar Impact Craters Based on Optical Data and DEMs Acquired by the Chang'E Satellites. IEEE Journal of Selected Topics in Applied Earth Observations and Remote Sensing. Vol. 8 (2015) No. 10, p. $4751-4761$.

[7]. Nur Diyana Kamarudin, Siti Noormiza Makhtar, Hizrin Dayana M, et al. Craters Detection on Lunar. IEEE International Conference on Space Science and Communication. Penang, Malaysia, 12-13 July 2011, p. 190-195.

[8]. Kun Li, Lingli Mu,Jianjun Liu, Chunlai Li. Impact crater detection based on regional segmentation using Chang'E-1 CCD data. 2011 4th International Congress on Image and Signal Processing. Shanghai, China, 15-17 October 2011, p. 1911-1915.

[9]. M.C. Burl, T. Stough, W. Colwell, et al. Automated Detection of Craters and Other Geological Features. Proceeding of the 6th International Symposium on Artificial Intelligence and Robotics \& Automation in Space. Montreal, Canada, 18-22 June 2001, p. 1-8.

[10]. JIANG HongKun, TIAN XiaoLin, XU AoAo. An automatic algorithm for detecting lunar impact craters in a defined feature space, SCIENTIA SINICA Physica, Mechanica \& Astronomica. Vol. 43 (2013) No. 11, p. 1430-1437. 